\title{
The Grounding of Computational Psychoanalysis:
}

\author{
A Comparative History of Culture Overview of Matte Blanco Bilogic
}

\author{
Giuseppe Iurato \\ Department of Physics \\ University of Palermo \\ Palermo, Italy \\ giuseppe.iurato@unipa.it
}

\begin{abstract}
In this paper, we wish to highlight, within the general cultural context, some possible elementary computational psychoanalysis formalizations concerning Matte Blanco's bi-logic components through certain very elementary mathematical tools and notions drawn from theoretical physics and algebra.
\end{abstract}

Keywords - bi-logic; groupoid; symmetry breaking; double bind.

\section{INTRODUCTION}

Ignacio Matte Blanco (1908-1995) was an outstanding psychiatrist and psychoanalyst who gave, among other things, notable contributions to the epistemological status of psychoanalysis starting from the Freudian theoretical framework. At the basis of his rigorous formulation of psychoanalytic foundations based on bi-logic, he put two main principles, namely the generalization principle and the symmetry principle. We refer to [1] for any further deepening of his thought. One of the central points of Matte Blanco thought is then the crucial transition from symmetric to asymmetric thinking. In this article, we want to make some formal remarks on the symmetry-asymmetry duality of Matte Blanco bi-logic, within the context of theoretical computational psychoanalysis. We think that Matte Blanco bi-logic is the most suitable one to be used for trying to employ a creative dimension from a computational standpoint, if one considers the unconscious as the main source of insight. Indeed, the basic inseparability between the symmetric logic and the asymmetric logic within the bi-logic context, might turn out to be useful just to this end. In this brief article, in particular, we would like to put into evidence some elementary formalizations which are offered as possible formal tools that may shed light upon the above mentioned link between symmetric and asymmetric logic. This is the chief point upon which we want to focus in the present paper. Within the general cultural context, we shall try to clarify Matte Blanco's intertwining of symmetric and asymmetric logic by means of certain very elementary mathematical tools and notions drawn from theoretical physics and algebra.

\section{THE BASES FOR A FORMALIZATION ATTEMPT}

\section{A. On Matte Blanco's bi-logic: a brief review}

The central point of Matte Blanco's bi-logic is the inextricable intertwining of symmetric logic, which reigns in the unconscious realm and is mainly regulated by the generalization and symmetry principles, and the asymmetric logic, which rules conscious thought. We are mainly interested in the passage from the former to the latter, this being that process which, if appropriately formalized, may turn out to be of some usefulness from a computational viewpoint ${ }^{1}$. In pursuing this, we follow a line of contextualization which includes some outstanding figures of the history of culture, amongst whom are Gregory Bateson, Claude Lévi-Strauss, André Weil and Robert R. Bush, as well as some elementary but basic formal tools and notions drawn from theoretical physics and algebra. Herein, we outline some main points of Matte Blanco's thought, following [2].

Matte Blanco's work has been centred around the new and ambitious intention of analysing unconscious logic through rational thought. To do this, he put two main principles at the basis of his framework, the generalization and symmetry principles, which are able to explain the main characteristics of the Freudian unconscious, namely displacement, condensation, absence of time, replacement of the external reality with the internal one, and absence of contradiction. Matte Blanco's work allows us to clarify the concept of consciousness. Starting from the paradigm of visual perception, based on the dual relationships between macular (or central) and peripheral vision, the focus on one object is perceivable only through a series of continuous eye pupil oscillations around it and not with a direct, fixed and central focusing on the object itself that will lead to an evanescence of the field of view. Analogously, consciousness, like eye movements, builds up asymmetric relationships around the object which is perceivable only through the latter, allowing to distinguish a thing from another. An object of consciousness is the result of a kind of bridling net of asymmetric relations ${ }^{2}$ built up around an emotional nucleus of symmetric relations. Consciousness, therefore, acts in an analytical manner, whereas general emotion, running

${ }^{1}$ In this paper, we mainly stress the passage from symmetric to asymmetric thought. Nevertheless, if one considers the symmetryasymmetry duality as, in a certain sense, analogous to the Freudian primary-secondary process duality, then it will be very interesting, above all from a computational psychoanalysis standpoint, to look at the general relationships between the elements of these dual pairs. In this regard, for instance, the reverse passage from the asymmetric logic to the symmetric one, presumably is domain of the repression, trauma and dream.

${ }^{2}$ A mental image, which often is prior to a definition, is also a set of relationships, hence an asymmetric thought outcome. 
symmetrically, acts in a global or synthetic manner. Nevertheless, both dimensions, or modes of being, are always inseparable and continuously interacting amongst each other. Psychoanalysis has pointed out the fundamental importance of emotion for the psychic life of every human being. The characters of consciousness may be detected only through introspection, which is an asymmetric phenomenon that concerns the time immediately prior to the introspection act itself. Hence, we may only have a retrospective introspection, because it is not possible to think something and, at the same time, to be aware of thinking. This is mainly due to the basic fact that, into the consciousness, not more than one asymmetric relation per time is presentable. Thus, the typical feature of rational thinking seems to be that of reflecting itself upon consciousness. In this sense, questions on psychological and physical time arise, reaching to touch philosophical issues concerning existentialism and phenomenology.

A pure sensation is something having an elusive and fleeting character, which can be caught by consciousness in an extremely brief time before it is inextricably harnessed into some implicit or explicit proposition (asymmetric relation). At a given instant of time, in the consciousness field there may be present only one phenomenon, the other ones going out from the field. Matte Blanco points out the primary fact according to which any human psychic act is roughly an inseparable pair made both by an emotional nucleus and by a surrounding rational thought component, with a variable ratio of reciprocal combination. The emotional component is made by symmetric relations, whereas the rational thought component is made by asymmetric relations. These two components are inseparable from each other, and in continuous reciprocal interaction. Analysing some types of emotions, like falling in love, fear and discouragement, it emerges that the main aspect of the symmetric relations characterizing emotion, is that each emotion refers not only to the intentional concrete object but to the wider and whole class to which such an object belongs, that is to say, to the equivalence class of which such an object is one of its representative elements. Indeed, when one, for instance, loves a person, this latter is seen as someone who goes on beyond herself or himself, personifying the attraction and representing all the attractive persons (maximization - see below). Therefore, three main features of the symmetric relations involved in a (strong) emotional status, are identifiable, namely: $i$ ) the generalization of all the properties of the intentioned object; $i$ ) the maximization of the properties of this object; iii) the transposition of this object to every other one who may be represented by it. All this recalls in mind the basic features of an algebraic equivalence relation. Then, the rational thought will build up a covering net made by asymmetric relations around such an emotional nucleus, made by symmetric relations, which constitutes the first and unavoidable apperceptive capture moment of the intentional cathexis object. The main consciousness activity is essentially analytic because it basically subdivides such a globally intentioned object (by emotion) into its constitutive parts, to give rise then to those asymmetric relations which will characterize the consciousness grasp of such an object ${ }^{3}$.

${ }^{3}$ This situation is paradigmatically similar to the essence of the socalled inverse scattering according to which a given non-directly
Symmetric and asymmetric modes of being are inseparable from each other because an utterly symmetric mode is a typical feature of psychotic or loss of consciousness states, whereas a complete asymmetric mode is also impossible since it would entail a total absence of any cathexis object, which is impossible because of the intentional nature of human desire. As we will see later, we shall try to formalize in an elementary way this last basic process of consciousness putting it into analogy with a formal process consisting of a kind of truly elementary (inversion) symmetry breaking of equivalence relations (of symmetry thought) into asymmetric ones (asymmetry thought). These very simple considerations may also be laid out within a wider and ambitious research program of a sort of psychoanalytic physics centred around the general relationships between primary and secondary psychic process, and whose early origins may be retraced in the Freudian Project for a Scientific Psychology ${ }^{4}$ (1895).

\section{B. From theoretical physics: on symmetry breaking}

Symmetry arguments are very important in natural sciences. These have also played a certain role in linguistics, starting from Noam Chomsky's formalization attempts up until recent linguistic invariant theory (see [4]). Following almost verbatim [5], the symmetry of the initial state of a given situation implies the complete equivalence between the existing possible alternatives (for example, the left bundle of hay with respect to the right one of Buridan's ass argument). If the alternatives are completely equivalent, then there is not a sufficient reason for choosing between them, the initial situation remaining unchanged if also the Leibnizean principle of sufficient reason (PSR) cannot be applied to settle the question. Arguments of this kind - that is, arguments leading to definite conclusions on the basis of an initial symmetry of the situation plus PSR have been used in science since antiquity. The form they most frequently take is the following: a situation with a certain symmetry evolves in such a way that, in the absence of an asymmetric cause, the initial symmetry is preserved. In other words, a breaking of the initial symmetry cannot happen without a reason, or an asymmetry cannot originate spontaneously. So, the notion of symmetry breaking in physics makes its appearance, which historically dates back to some works made in 19th century solid state physics.

To be precise, the study of symmetry breaking goes back to Pierre Curie, who formulated three principles about crystal symmetry on the basis of some of his notable crystallographic research made in the 1890s. Following [6], he was primarily concerned, not with the symmetry of the dynamical equations, but rather with that of their solutions, i.e. with the symmetry of

observable physical object is indirectly detectable only through the set of asymmetric relations emerging from it (like those forming the scattered field emerging from the scattered object). This resembles a metaphoric explanation of what scattering is, due to P.T. Matthews (1919-1987) (see [3, Chapter 10]), according to which the essence of the scattering methods is like to determine the form of an invisible statue (i.e., the mathematical form of the scattering potential) from the angular distribution and intensity of the emerging rays due to a watering of it.

${ }^{4}$ For instance, when, in Chapter 1, Section 3 of this work, Freud talks about contact-barriers, we may identify a certain conceptual analogy with the potential wells of elementary quantum mechanics. 
the physical states. This phenomenological approach led him naturally to emphasize the role of asymmetry rather than that of symmetry. A symmetry can be exact, approximate or broken. Exact means unconditionally valid; approximate means valid under certain conditions; and broken can mean different things, depending on the object considered and its context. According to Curie, symmetry breaking has the following role: for the occurrence of a phenomenon in a medium, the original symmetry group of the medium must be lowered (broken, in today's terminology) to the symmetry group of the phenomenon (or to a subgroup of the phenomenon's symmetry group) by the action of some cause. In this sense symmetry breaking, or asymmetry, is what creates a phenomenon. Following [6], Curie was in a better position to appreciate the role of symmetry breaking as a necessary condition for the existence of phenomena. Generally, the breaking of a certain symmetry does not imply that no symmetry is present, but rather that the situation where this symmetry is broken is characterized by a lower symmetry than the original one. In group-theoretic terms, this means that the initial symmetry group is broken into one of its non-trivial subgroups. It is therefore possible to describe symmetry breaking also in terms of relations between transformation groups, in particular between a group (the unbroken symmetry group) and its nontrivial subgroups. As has been clearly illustrated by Stewart and Golubitsky (see [7]), starting from this point of view, a general theory of symmetry breaking can be developed by tackling such questions as which non-trivial subgroups can occur?, when does a given subgroup occur? See also [7] and the next section. The notion of symmetry breaking is emblematic to explain the paradigmatic dialectic contraposition between symmetry and asymmetry.

Symmetry breaking was first explicitly studied in classical physics with respect to physical objects and phenomena. This is not surprising, since the theory of symmetry originated with the visible symmetry properties of familiar spatial figures and everyday objects. As has been said above, it was Curie's works on crystal symmetry that opened this new perspective. However, it is with respect to the laws that symmetry breaking has acquired special significance in physics. There are two different types of symmetry breaking of the laws, explicit and spontaneous, the case of spontaneous symmetry breaking being the more interesting from a physical as well as philosophical point of view. Explicit symmetry breaking (ESB) refers to a situation where the related dynamical equations are not manifestly invariant under a certain symmetry group. This means, in the Lagrangian or Hamiltonian formulation, that the Lagrangian or Hamiltonian operator of the system contains one or more terms (like anomalies) which are explicitly breaking the symmetry. Spontaneous symmetry breaking (SSB) instead occurs in a situation where, given a symmetry of the equations of motion, solutions exist but are not invariant under the action of this symmetry often without any explicit asymmetric input ${ }^{5}$

5 In this regard, [8] states that in the development of theoretical physics, the standard way of describing a broken symmetry has been that of introducing an explicit non-symmetric term in the equations of motion. A real revolution occurred with the realization of a much more economical and powerful mechanism, called spontaneous symmetry breaking, by which symmetry breaking may be realized (hence the attribute 'spontaneous'). A situation of symmetry breaking can be first illustrated by means of simple cases taken from classical physics: an emblematic and meaningful example is given by the phenomenology of the well-known Euler's elastic bar. In this case, the actual breaking of the symmetry may then easily occur by effect of a (however small) external cause, and the stick bends until it reaches one of the infinite possible stable asymmetric equilibrium configurations. In substance, what happens in the above kind of situation is the following: when some parameter reaches a critical value, the lowest energy solution respecting the symmetry of the theory ceases to be stable under small perturbations and new asymmetric (but stable) lowest energy solutions appear. The new lowest energy solutions are asymmetric but are all related through the action of the symmetry transformations (which are asymmetric relations in the Matte Blanco sense). Therefore, it seems that for reaching equilibrium solutions of a given dynamically unstable problem, it is needed to break the initial symmetry of unstable and unrelated states, so obtaining solutions with less symmetry (asymmetry) but more stability; furthermore these latter equilibrium states, meant as asymmetric solutions of the given dynamical problem, are related among each other by (asymmetric) relations given by the action of a transformation group. In quantum physics, instead, SSB is applicable only to infinitely extended systems (like unconscious, according to Matte Blanco). These last considerations might also turn out to be useful in regard to further formal considerations about an object's relationship.

Historically, the concept of spontaneous symmetry breaking first emerged in the condensed matter physics of the first half of 20th century, and was later transferred to quantum field theory (QFT) in the 1960s, above all in relation to weak interactions. The notion of symmetry breaking still waits to be laid out within a unified formal treatment because of its variegated nature and the different contexts in which such a notable mechanism is involved. Herein, we shall consider only the quantum context in which it is better known and studied from a formal viewpoint. The spontaneous breakdown of a global continuous internal symmetry gives rise to massless bosons (known as Goldstone bosons) according to a general QFT theorem, known as Goldstone theorem, stated by J. Goldstone in the early 1960s and valid only in the case of global continuous symmetries; moreover, other important elements are locality and infinite dimensionality (see [8]). Subsequently, starting from the previous notion of dynamical symmetry breaking (DSB) related to the creation, via Higgs mechanisms, of massive gauge vector bosons from symmetryviolating vacuum expectation values of Higgs scalar fields, a more general mechanism was proposed, today also known as the Englert-Brout-Higgs-Guralnik-Hagen-Kibble mechanism (hereafter briefly called Higgs phenomenon), according to which, when a global internal gauge symmetry is promoted to a local gauge symmetry, then Goldstone bosons disappear and gauge bosons acquire mass, this taking place often without explicitly breaking the gauge invariance of the theory. Following [8], in the Haag-Kastler algebraic approach to

even if the equations of motion are symmetric. Furthermore, [8] points out that there also exist cases in which asymmetric inputs are needed. 
quantum field theory, the essence of a spontaneous symmetry breaking for an infinitely extended system, with a locality condition related to the algebra of local fields (i.e., local operators) $\mathcal{F}$ which, in turn, contains, as subalgebra, the $\mathbb{C}^{*}$ algebra of observable fields $\mathcal{F}_{\text {obs }}$ associated with the given system, is as follows. From the pioneering works by E.P. Wigner, the physical observables of such an algebra can be obtained only through a suitable Hilbert space representation $\pi$ of $\mathcal{F}_{\text {obs }}$. There is a symmetry group $G$ acting on a ray Hilbert space $\mathcal{H}$ to give a group of unitary operators $U(g)$ of the latter. This gives rise to a group of algebraic automorphisms of $\mathcal{F}_{\text {obs }}$ through the algebraic map $g \rightarrow \alpha_{g}$ given by $\alpha_{g}(A)=$ $U(g) A U(g)^{-1}$ for each $A \in \mathcal{F}_{o b s}$, which induces an action of the group $G$ on $\mathcal{F}_{\text {obs }}$ via the assigned Hilbert space representation $\pi$, in turn induced by the group of unitary operators $\{U(g) ; g \in G\}$ via the Wigner's theorem. Nevertheless, in the case of an infinitely extended system, there may be automorphisms $\alpha_{g}$ which are not described by unitary operators provided by a representation $\pi$ of $\mathcal{F}_{\text {obs }}$, that is to say, $g$ exists as a symmetry at the algebraic level, but it is not a symmetry of the realization of the system provided by the representation $\pi$ in $H_{\pi}$, that is, it is not implemented by unitary operators in $H_{\pi}$. In this case, we say that the symmetry $g$ is broken in $H_{\pi}$. Therefore, there is a substantial level detachment between the algebraic and the functional stance of a symmetry when this is broken. This fact, that is to say disjoint realizations of the physical system induced by inequivalent representations of $\mathcal{F}_{\text {obs }}$, is the main essence of the mechanism of symmetry breaking. It is just this inequivalence of, at least, two distinct representations to give rise asymmetry, for infinitely extended systems: in this case, the breaking of color symmetry mentioned in [9] and made possible thanks to the comparison with the environment or background, is very meaningful to clarify the idea. From quantum cromodynamics case studies, it is also known that highest symmetry levels implies a flattening of reality, so that asymmetry - arising from symmetry breaking - is a fundamental element to detect this, as well as to account for diversity.

As has already been said above, symmetry breaking raises a number of deep philosophical questions, such as the one asking about the evidence for the (hidden) symmetry underlying the directly observable asymmetry. SSB allows symmetric theories (like those concerning unconscious) to describe asymmetric reality (of consciousness), assuming the salient fact that an observed asymmetry requires the action of a cause which can be an explicit breaking of the symmetry of the laws or asymmetrical initial conditions or SSB. This last consideration is very similar to the aim of the Curie principle that, when extended to include the case of SSB, is substantially equivalent to the methodological principle according to which an asymmetry of the phenomena must come from the explicit or spontaneous breaking of symmetry of fundamental laws. This might be called an extended Curie principle. Following [9], roughly we might say that the general mechanism of spontaneous symmetry breaking related to a physical system is characterized by symmetric interactions between its parts, but the environment in which the system stays is not symmetric (like the vacuum), this implying an asymmetric behaviour of the above interactions. In general, the lowest energy states lie at the bases of this asymmetry. However, in agreement with [9], it is just the comparison between, at least, two different realizations that highlights the paradigmatic duality symmetryasymmetry through the symmetry breaking mechanism, this being just one of the possible philosophical aspects inherent the general symmetry breaking phenomena, to which we shall refer later.

\section{From mathematics: on groupoids}

The groupoid structures have a wide range of applications, ranging from pure and applied mathematics to physics and computer science. We refer to [10], [11], [12] and [13] for more information. In what follows, we are interested in a particular groupoid structure which is the most suitable one to formalize in an elementary way what is our object of discussion. Roughly speaking, a groupoid is simply an algebraic system with a partial binary relation. A more precise but succinct definition is that a groupoid $G$ is a small category in which every morphism is an isomorphism. Thus $G$ has a set of morphisms, which we shall call just elements of $G$, a set $O b(G)$ of objects or vertices, together with two functions, say $s, t: G \rightarrow O b(G), i: O b(G) \rightarrow G$ such that $s i=t i=1$. The functions $s, t$ are sometimes called the source and target maps respectively. If $a, b \in G$ and $t a=s b$, then a product or composite $a b$ exists such that $s(a b)=s a, t(a b)=t b$. Further, this product is associative; the elements $i x, x \in O b(J)$, act as identities; and each element $a$ has an inverse $a^{-1}$ with $s\left(a^{-1}\right)=t a, t\left(a^{-1}\right)=s a, a a^{-1}=i s a, a^{-1} a^{-1} a=i t a$.

An equivalence relation $R$ on $X$ becomes a groupoid with $s, t: R \rightarrow X$ the two natural projections, and product $(x, y)(y, z)=(x, z)$ whenever $(x, y),(y, z) \in R$. There is an identity, namely $(x, x) \in \Delta(R)$, for each $x \in X$. A special case of this groupoid is the coarse or natural groupoid $X \times X$, which is obtained by taking $R=X \times X$. This apparently banal and foolish example is found to play instead a key role in the theory and applications. At the opposite extreme to the coarse groupoid $X \times X$, is the fine groupoid on $X$ that can be considered as the diagonal equivalence relation on $X$, or alternatively as the groupoid $X$ consisting only of identities, namely the elements of $X$. This consideration of an equivalence relation as a groupoid also suggests the utility of groupoids for studying quotienting constructions, particularly in cases where the quotient set $X / R$ cannot carry the appropriate structure. We are just interested in these last types of groupoid structures. Following [14], given any set $X$ endowed with an equivalence relation $\sim$, we define a groupoid structure over $X$ by letting $\mathcal{G}_{\sim}=\left\{(x, y) \in X^{2}: x \sim y\right\}$ together with the following multiplication

$$
\begin{gathered}
\circ:\left\{((x, y),(y, z)) \in \mathcal{G}_{\sim}^{2} ;(x, y, z) \in X^{3}\right\} \rightarrow \mathcal{G}_{\sim}, \\
((x, y),(y, z)) \rightsquigarrow(x, z) .
\end{gathered}
$$

This operation is well-defined by the transitivity of the equivalence relation, which also gives the associativity property. Now, the symmetry of $\sim$ ensures that for all $(x, y) \in$ $\mathcal{G}_{\sim},(y, x)$ is also in $\mathcal{G}_{\sim}$, the latter being clearly the inverse of the former. Finally, the reflexivity is not required to define the structure; the set of units of this groupoid is the set of elements $x$ of $X$ such that $x \sim x$, so when $\sim$ is reflexive, then $\mathcal{G}^{0}=$ 
$\{(x, x): x \in X\} \cong X$. Note that, for any $(x, y) \in \mathcal{G}_{\sim}$, $s((x, y))=x$ and $r((x, y))=y$, whilst $(x, y)^{-1}=(y, x)$. We shall name this groupoid the groupoid graph of the relation $\sim$. Also [11] considers groupoids as generalized equivalence relations.

\section{SOME ELEMENTARY FORMALIZATION ATTEMPTS}

\section{A. Again on groupoids}

The above mentioned groupoid structures will make possible certain formalization attempts of some main aspects of Matte Blanco's bi-logic. The groupoid structure has been proved to be at the early bases of almost all the most basic commutative and non-commutative algebraic structures, so that they lie at the deeper roots of the general algebraic formalization. In particular, groupoid structures are also at the basis of graph and combinatorial structures (see [15] and references therein) as well as having applications in type theory (see [16]); likewise, ordered groupoids are at the foundations of other algebraic structures, like groups and inverse semigroups (see [17]). Finally, groupoids have recently received remarkable attention also in non-linear dynamics of networks: in this regard, see [7], where a very interesting discussion of network synchrony, asynchrony and related symmetry breaking phenomena, in the context of groupoid formalism, is made ${ }^{6}$. The main feature and potentiality of a groupoid structure is just the partiality of its binary operation, that makes this structure quite versatile.

On the other hand, following [4], for some time mathematicians have argued for the more general groupoids as more suitable structures for treating symmetries. At the crossroads between groups and groupoids, lies a distinction between local and global symmetries. For physicists, the localglobal symmetry distinction is aligned with the distinction between local and global transformations. For mathematicians, the distinction is based on whether part or the entire structure is conserved (the former requiring a groupoid and not a group representation). Therefore, groupoids are offering more flexible mathematical structures embracing a wider formal tool box to treat symmetry and related phenomena. Groupoids can be understood not only as generalized groups but we can also see them as generalized equivalence relations: to be precise, it is possible to prove the existence of a certain two-way correspondence between groupoids and equivalence relations: see [4] and [11] for more details. Meant as a generalization of equivalence relations between parts of an object, groupoids open the door to local symmetries. The group, instead, can only represent global symmetries, that is to say it is unable to put into relation parts of those (whole) objects which are involved in the group transformations. Groupoids will be those unifying formal structures which will comprise either local and global symmetries, both from physical and mathematical standpoint (see [11]). Thus, since we stress the fact that equivalence relations, according to Matte Blanco, characterize the unconscious, it is immediately realized that groupoids are the most suitable elementary formal structures to try to give a minimal theoretical framework of this psychoanalytic construct.

6 As regard possible links between Matte Blanco thought and synchronous/asynchronous logic, see also [18].
Finally, we consider ordered groupoids, that is to say groupoids equipped with a compatible partial order relation. Following [19], every ordered groupoid is isomorphic to one constructed from a category acting in a suitable fashion on a groupoid arising from an equivalence relation. Lawson considers the simplest groupoids, those arising from equivalence relations and named combinatorial groupoids. Then, he constructs ordered groupoids from combinatorial ones plus some data, namely a partial order in turn inferred from a partial preorder relation that, quotienting, gives rise to a partial order. In turn, such a preorder relation may be induced by a suitable category action on the given combinatorial groupoid. Thus, the essential steps are as follows:

- A category $C$ acts on a combinatorial groupoid $H$.

- It induces a preorder $\preccurlyeq$ on $H$ whose associated equivalence relation is $\equiv$.

- The quotient structure $H / \equiv$ is a groupoid $J(C, H)$ on which the preorder induces an order.

- The groupoid $H / \equiv$ is ordered and every ordered groupoid is isomorphic to one constructed in this way.

This is the clear formal treatment given by Lawson, in which a final universality property is proved, namely, every ordered groupoid is isomorphic to one of the form $J(C, H)$ for some action of a category $C$ on a combinatorial groupoid $H$. Roughly, if the above action of $C$ on $H$ is of the general type $(a, x) \rightarrow a \cdot x$ (e.g., it may provide some interpersonal relation) then we put $x \leqslant y$ in $H$ if and only if there exists $a \in C$ such that $x=a \cdot y$, being then easy to prove that $\preccurlyeq$ is a preorder; then, $x \equiv y$ if and only if $x \leqslant y$ and $y \leqslant x$, is an equivalence relation on $H$. Finally, an order on $H / \equiv$ is given by setting $[x] \leq[y]$ if and only if $x \leqslant y$, that is to say, if and only if there exists $a \in C$ such that $x=a \cdot y$, this last definition turning out to be well-posed. Finally, given $O(x)=\{a \cdot x ; a \in C\}$ the orbit of $x$ under the given action of $C$ on $H$, then it is easy to prove that $[x]<[y]$ if and only if $y \notin O(x)$, that is to say $O(x) \cap$ $O(y)=\varnothing$, with a consequent violation, with respect to the given action, of the inversion symmetry $(x, y) \rightarrow(y, x)$, which is the simplest example of a permutation symmetry breaking with respect to the diagonal. Therefore, a strict order arises from a simple inversion symmetry breaking, which basically expresses an asymmetry condition as a simple outcome of the inequivalence of at least two different representations. Furthermore, following [20, Chapter 2], every transitive action of a group/groupoid structure $G$ on a set $M$ induces on it a homogeneous space structure. It is possible to prove that the study of homogeneous spaces can, in principle, be reduced to the study of coset spaces $G / H$ when $H$ is a sub-group of $G$, hence to the study of pairs $(G, H)$ of the type group/groupoidsubgroup/subgroupoid, just the typical formal pair of algebraic structures involved in the above formulation of symmetry breaking phenomena. On the other hand, a strict ordering is, in a certain sense, a formal condition characterizing the asymmetry involved in every possible hierarchical structure which is a strictly ordered structure too. In conclusion, we can say that, in a certain sense, inversion symmetry breaking is a compatible mechanism that, for instance, via combinatorial groupoid or groupoid graph structure, is offering to formalize 
the passage from symmetry (essentially represented by an equivalence relation) to asymmetry (essentially represented by a strict order relation ${ }^{7}$ ). As has been said at the end of section II.C, a groupoid structure is naturally associated with every equivalence relation, where reflexivity is not necessarily required. Now, the properties of an equivalence relation approximately correspond, by means of a kind of cryptomorphism theorem, to Matte Blanco's generalization ${ }^{8}$ and symmetry principles, whereas a preorder is simply a reflexive and transitive binary relation. An order [strict order] is an antisymmetric preorder that differs from an equivalence relation simply replacing the symmetry condition with an antisymmetric [asymmetric] one. Thus, with a breaking of inversion symmetry, we obtain an asymmetric relation. Therefore, via groupoid graph or combinatorial groupoids, it is possible to go from an equivalence relation to an order one, basically through a suitable inversion symmetry breaking.

\section{B. On the formal structure of kinship, on the Gregory Bateson double bind, and all that}

Following [21], it was André Weil and Robert R. Bush to give, starting from Murngin system, a first attempt to formalize kinship by means of permutation group structures, exposed in a very interesting appendix to chapter XIV of the celebrated Claude Lévi-Stauss 1949 work Les Structures Élémentaires de la Parenté; see also [22]. While the empirical cultural content remains to be filled in, the problem of formal representation of kinship is concerned with the relations or orderings between culturally given unions such as reproductive or other types of matings, not the cultural characteristics of the unions themselves. In graph theoretic terms, vertices and their labels, even while associated, are distinct. Therefore, ordered structures are at the early bases of every further attempt to formalize kinship. After this work of Weil and Bush, other formalizing attempts were attained by other scholars, mainly using graphs and other ordered structures. But, having seen that groupoids are at the basis of all these just mentioned structures and having seen too that not all binary relationships are allowed in any possible kinship structure theory, it seems quite natural to think groupoids (like combinatorial groupoids or groupoid graphs) as the most suitable structures to formalize kinship and, in general, interpersonal relationships, just due to partiality of its binary operation. In any case, every possible formal structure used so far to formalize kinship, is an a priori given ordered structure which may be thought as a formal framework of a social structure as it really appears, without

${ }^{7}$ Furthermore, the strict set inclusion is a (partial) strict order which allows to distinguish, for instance, between set and (proper) subset, hence to introduce a minimal hierarchical setting.

${ }^{8}$ Matte Blanco generalization principle shouldn't be literally meant as it is commonly stated, because its usual definitions could lead to the wrong possibility to clearly distinguish between set and subset in a hierarchical way, a thing which is impossible to do in the unconscious realm. Instead, such a generalization principle should be rather understood as expressing the ability to make equivalence classes and partitions. Only overcoming symmetry and generalization principles, it will be possible to reach communicative metalevels, the simplest of which having been those concerned with the various distinctions among element, subset, set and class arose, with the rising of type theory. giving any hint on the possible early origins of it, that is to say, how and whence it came about. On the other hand, according to [23, Model 2], thanks to the Weil and Bush work, the matrimonial rules of primitive societies are aimed to hinder the marriage between close kin even in the case in which the involved individuals weren't aware of being in a kin relationship; this was possible because there exist primitive ${ }^{9}$ societies where familiar links are liable to be quickly forgotten. This means that implicitly (or unconsciously) they follow just these group rules, so that it is allowed to consider group/groupoid structures as tools suitable to formalize such questions, in a very precise manner. Not all marriage types are allowed, the marriages between brother and sister turning out to be automatically hindered, so that it is as if Oedipal rules unconsciously acted to shape kinship ${ }^{10}$. This is a notable fact because it is very amazing to see how a primitive society, through trial and error, has been able to perform formal structures of a certain complexity, like the group ones. Then, since psychology (see [24]) and social anthropology (see [25]) are nowadays even more oriented to consider a familial nucleus as the primary structural key of society as well as the natural environment that much more influences the psychological growth of an individual, in agreement with this, we would want to put forward the hypothesis that the familial triadic structure is the key component which lies at the early basis of any possible further social formal structure in agreement with what Lévi-Strauss himself states in [25]. Therefore, we want to focus on the family structure, believing that it is just its deep triadic structure that mostly contributes to the individual's basic psychological formation.

Now, according to Matte Blanco, the main feature of consciousness is just the passage from the symmetric logic to the asymmetric one, so that it is of extreme importance to shed light on this crucial step. The minimal cardinality of a nontrivial ordered groupoid is three for two chief reasons: first, to warrant a non-trivial transitive property, second because of the fashion with which an ordered groupoid is obtainable, that is to say by means of non-trivial action of a non-trivial category with at least two elements, on a non-trivial combinatorial groupoid with at least two elements. Now, in the 1950s, Gregory Bateson, Don D. Jackson, John Weakland and Jay Haley gave a possible (interpersonal) interpretation of schizophrenia based on the so-called double bind (see [26, Part III]), in which the familiar nucleus plays a fundamental role. Let us briefly discuss it. Bateson, like Matte Blanco, knew very well Bertand Russell and Alfred Norton Whitehead type theory, the first one that enabled to identify different levels of abstraction, pointing out the criticalities and potentialities of two crucial axioms of formal set theory, the axiom of specification and the axiom of extensionality, which allow to clearly distinguish between element, set and class, starting from Cantor naïve set theory. So, the way towards

\footnotetext{
${ }^{9}$ The primitiveness condition is a primary working hypothesis which puts us closest to unconscious behaviour.

${ }^{10}$ This is coherent with what Lévi-Strauss claims about the origin of culture and society (hence consciousness), because he puts the Oedipus complex at the basis of the crucial passage from nature to culture. Moreover, from this standpoint, we might also identify a kind of anthropomorphic origin of group structures.
} 
metamathematics had been opened. Taking into account this theory, as well as previous notable studies made by Rudolf Carnap, Ludwig Wittgenstein, Frieda Fromm-Reichmann and Benjamin Lee Whorf, first Bateson considered the possibility of the existence of many abstraction levels in human communication. For instance, in schizophrenia, there is often a confusion between literal and metaphoric levels of either her or his own and other messages (inability to metacommunicate). $\mathrm{He}$ also argues on the lack of the main formal properties characterizing an equivalence relation, that is to say reflexivity, symmetry and transitivity, which are almost totally disregarded by primary process ${ }^{11}$. There, indistinguishable are everyone from someone and no-one, as well as the whole from the part, which are features of conscious processes. Then, Bateson and co-workers point out the fact that a schizophrenic is mainly unable to contextualize (to realize metacommunicative frameworks, according to Bateson), that is to say, he or she has serious problems in semantics and pragmatics tasks ${ }^{12}$, as well as in discriminating the various logical types. Bateson and coworkers bring this back to the presence of a double bind. The double bind will be retaken by the Palo Alto school of Paul Watzlawick on pragmatic communication (see [28]).

The notion of double bind is quite original, and refers to the presence of a double and ambiguous link between mother and daughter/son which moulds interpersonal relations centred around an emotively ambiguous and contradictory communication between mother and daughter/son, and that will engrave, in a negative manner, upon the logical abilities of the girl or boy, hindering the capacity to distinguish the logical status of the thoughts, if the paternal figure does not act as an intermediary $^{13}$, a kind of "breaker" coherently with what will be said later. Indeed, it is as if such a paternal figure acted by breaking the inversion symmetry between mother and daughter/son, re-establishing the right hierarchical position within the familial nucleus. The paternal figure acts to make inequivalent at least those two primary representations given by maternal and paternal figures, as a kind of symmetry breaking, according to what has been said in section II.B. The double bind plays a fundamental role in structuring the family. Indeed, following [30], from the clinical analysis of the triadic familial structure of six female patients, it turns out that in all these cases but a psychotic patient, the mother permitted a third person - like father - to be present between the double bind given by mother-daughter, acting as a sort of "role inverter": in fact, Jackson reports, for instance, that the father's closeness and overt interest in his daughter, was the reverse of the mother's in that it tended to decrease as the child grew older and, in some instance, was abruptly terminated at the

${ }^{11}$ For instance, in [27, pp. 40-44], it is reported the case of the intransitivity of psychological preferences, a kind of psychological basis for the well-known Kenneth Arrow theorem on social choices. See also [13] and references therein, about other interesting remarks on transitivity or not, concerning many examples of interpersonal relationships.

${ }^{12}$ Modern neurophysiologic research confirms this last fact, that is, schizophrenics have mainly impaired the basic semantic-pragmatic integration functions (see [29] for a very brief review).

${ }^{13}$ In this, recalling the famous Lacanian name-of-the-father (see [29]). menarche. These patients have been trained symbiotically to feed on triadic involvement. This is most apparent when they are interacting with only one person and must in fantasy involve a third as though they feel no "ego wholeness" without a collection of "part egos". One price paid for this need to interact in two directions at the same time is a multifaceted inferiority feeling. For example, these women equate head and penis, hence intellect and maleness. The parental interaction constituted a nidus for the development of the girl's hysterical and phobic symptoms and acting out. Therefore, the paternal figure could be seen as acting ${ }^{14}$ on the double bind to break the often dangerous inversion symmetry mother-daughter/son ${ }^{15}$, distinguishing between, at least, two different realizations, so giving rise to an order relation; as regard symmetry of double bind, see [28]. In agreement with Matte Blanco's bi-logic, triadicity, therefore, is a primary and essential structural element to have the primary presuppositions for a normal psychic development. There exists literature on the relationships between Matte Blanco and Bateson. The above supposition concerning symmetry breaking made by the paternal figure is supported by the interesting work of Klaus Fink [33] about the bi-logic perception of time. Fink reports a clinical case of a young man (John) who had not a really present paternal figure but a dominant, incorporating and prevaricating maternal figure who caused a spread of symmetrical thought which entailed, amongst other things, a distortion of time perception, with an impairment of time ordering. Only through the transference intervention of the psychoanalyst, it was possible to try to restore a stronger paternal role, so recovering the right time perception, hence stemming maternal symmetric thought drives. John gradually re-acquired his own sense of individuality and awareness, a right time perception, a normal balanced relationship between physical and psychological time ${ }^{16}$, all elements, these, which

14 For a technical treatment of the theory of symmetry breaking via group action, see, for example, [31] as regard graph theory, and [32] as regard bifurcation symmetry breaking theory.

15 On the symmetry or reciprocity character of double bind, as well as on other interesting remarks and considerations, see [28,6.43] and references therein. In this work, the general presence and relevance of double bind, as well as the need for overcoming it, in regard to all those systems having a certain intelligence autonomy (including animals), is highlighted.

${ }^{16}$ In this regard, it would also be possible to suppose that the crucial passage from symmetric to asymmetric thought might be related to the equally crucial relationships between physical time (chrónos) and psychological time (kairós), amongst which there is no a priori twoway correspondence. Psychology of time, from Paul Fraisse onward (see [34]), says that these last two are independent amongst them. Perhaps, the acquisition of physical time perception, considered having an ontological dimension, might exert, according to Klaus Fink, a structuring action on psychological time, making easier or even inducing the passage from symmetric to asymmetric thinking. Fink's work has been confirmed by further studies on time distortion in the transference (see [35]), supporting the idea that psychic reality is strictly influenced by physical time, as experienced in psychoanalytic transference that, as known, above all reproduces the childish Oedipus conflicts, re-evoking the various familiar images. En passant, we remember that also the mathematician L. Brouwer claimed the primary importance of a sort of (Kantian) temporal apriori in the mathematical reasoning, that is to say, he gave much 
gave rise to a right phenomenological psychology relationship between here-and-now and there-and-then, roughly between psychic (or internal) and external reality. On the other hand, also Bateson and co-workers had already pointed out about the possibility to implicitly change rules only under transference setting ${ }^{17}$. Therefore, according to Fink, a correct reality-testing just consists in putting (also by means of transference intervention) the right relationships between symmetrical and asymmetrical thinking. Again according to Fink, the resolution of psychic conflicts has been considered as a sort of catastrophic point (also in the René Thom sense) by W. Bion, as well as an uncanny point by Freud himself, to highlight the criticality of the turning-point element marking the equally crucial transition point from symmetrical to asymmetrical thought. Fink's considerations also justify our view of this passage as a kind of symmetry breaking development. On the other hand, Freud too considered time as one of the main features of the passage from primary to secondary process, related to delaying of desire's satisfiability.

Taking into account what has been said in section II.B, if one considers the passage from symmetric to asymmetric thought as due to a kind of inversion symmetry breaking, besides that as an overcoming of Bateson and co-workers double bind mother-daughter/son through father action, then it is possible to account for the rising of ordering, hence hierarchical structures. In particular, taking into account what has been said about Higgs mechanism, the SSB allows the transition from a global to a local symmetry with a generation of massive bosons (asymmetry), which might be interpreted as an unavoidable presence of symmetry interstices into the asymmetry, a thing quite analogous to the bi-modal presence of symmetric and asymmetric thought. Therefore, although a global symmetry has broken into local symmetries, these latter never utterly disappear (because symmetry breaking in any case involves non-trivial groups), consistent with Matte Blanco according to whom conscious thought may include only few asymmetrised pieces of symmetry. On the other hand - see for instance [37] and [38] - at the early origins of space and time and their difference, there could be basically symmetry breaking phenomena. The unavoidable presence of symmetry interstices into the asymmetry might also be put into comparison with the so-called paraconsistent logics in which not all contradictions (due to symmetric thought) of a formal system have serious implications or relevance for the whole system; these contradictions may be relegated into neighbourhoods, or

attention to the basic role played by the temporal dimension. Likewise, some remarkable phenomenological-existentialistic trends of philosophy pay much attention to the role played by temporal dimension from a psychic standpoint. Finally, time perception seems to be impaired in schizophrenic patients (see [46]).

${ }^{17}$ And this could reflect also on Krikpe-Wittgenstein paradox and on a clarifying psychoanalytic revisitation of it. Following [36, I], Kripke provides a kind of collective solution to this paradox, that is to say, the reason (even if the usage of this term is paradoxical just in this context, unless we distinguish between implicit and explicit reasons - in this case, it would be better to speak of an implicit reason which will become explicit at the consciousness level) why we follow a rule should be a collective fact, not an individual one, as if it belonged to a sort of collective unconscious (like, for instance, the one described by C.G. Jung and C. Lévi-Strauss). circumscribed regions, in such a way that they cannot influence the coherence of the system, without having trivializations of it. Thus, also in logic, with this last type of confinement operations, it is possible, as the saying goes, to conceive a kind of contemporaneous presence of symmetric and asymmetric thought elements, coherently with Matte Blanco bi-logic.

\section{Other formal remarks}

Following the Klaus Fink work [33], we have pointed out the primary role played by transference intervention in setting up the right ratio between symmetric and asymmetric thinking. Also Bateson and co-workers pointed out the great force exerted by transference to implicitly change interpersonal rules which are the symbolic transposition of the familiar ones. The two main dimensions which characterize the transference, where the essence of childish Oedipus complex is re-evoked, are the actualization of past experience and the displacement towards the paternal figure. The paternal imago, in general, is the one that has major load in the transference intervention; other imago, like the maternal one, may also be involved in transference setting. Therefore, following Bateson and co-workers, we agree with the assumption that the paternal figure plays a very fundamental role in establishing the right interpersonal relationships (according to Freud, symbolically moulded on the familial ones) and in overcoming the pernicious double bind.

In the previous sections, we have tried to formalize, in an elementary fashion, the crucial passage from symmetric to asymmetric thinking, taking into account some elementary notions drawn from theoretical physics, and certain basic structures drawn from algebra. In particular, we have considered some groupoid structures which are offered to formalize certain notable aspects of Matte Blanco's bi-logic. Now, recent research on computational psychoanalysis made by Lauro-Grotto (see [39]) and Murtagh (see [40] and references therein) about structure of the semantic field, have realized that the main aspects of the unconscious realm, via Matte Blanco bi-logic, can be formalized by means of ultrametric structure and that the passage from symmetric to asymmetric thinking might be put into analogical comparison with the formal passage from ultrametric to metric. Khrennikov (see [41], [42] and [43]), then, has extended padic dynamical systems to mathematical modelling of mental space. To be precise, an ultrametric is a metric which satisfies a stronger triangular inequality, namely the following

$$
d(x, y) \leq \max \{d(x, z), d(z, y)\}, \forall x, y, z .
$$

Now, Lauro-Grotto claims that if one considers, following Matte Blanco, an ultrametric space model of unconscious, due to the intrinsic properties of such a type of formal space, there follows a notable restriction of semantic field because of the great number of clusters of concepts that become indistinguishable due to a loss of homogeneity in their inner structure, with a consequent loss of hierarchical ordering. Therefore, the Matte Blanco symmetric-asymmetric thinking duality could also be stigmatized by the formal duality 
ultrametric-metric. On the other hand, the inseparability of these last two notions, essentially given by the various isometric embedding theorems of ultrametric spaces into a Euclidean space (see [40] and references therein) could refer to the inseparability between symmetric and asymmetric thinking within the Matte Blanco framework. Finally, from this standpoint, Lauro-Grotto also claims the possible usefulness of the consideration of the replica symmetry breaking concept of spin glass theory, coherently with what has been said above about symmetry breaking phenomena. Moreover, the above considered groupoid structures might also be usefully and suitably implemented by these last metric structures to reach a most complete and general formal framework of computational psychoanalysis modelling the unconscious construct.

We conclude with a final but important remark. It could seem quite contradictory to use an order relation, like $\leq$, in defining a property that holds in the unconscious realm, such as the one expressed by the above ultrametric triangular inequality. Indeed, just in the unconscious realm, due to symmetry principle, it is unconceivable to think any order relation. But, again following Klaus Fink's work (see [33] and references therein), such an apparent contradiction may be easily clarified. In fact, as said above, in transference, it is possible to re-establish the right relationship between external and internal reality through the institution of the right correspondence between external and internal time perception. This was possible just thanks to transference action that allowed the patient to make him aware how something conceived symmetrically ${ }^{18}$ (unconscious disorder) must be judged asymmetrically ${ }^{19}$ (usage of an order relation $\leq$ in the ultrametricity condition); there are no other ways to do this ${ }^{20}$ because, having seen the nature itself of the unconscious, it couldn't be otherwise; according to Fink, this is ultimately what a reality-testing should consist of. On the other hand, the Matte Blanco epistemological program consists just in searching to explain unconscious phenomenology by means of rational thought. Therefore, in this only apparently contradictory sense, the Lauro-Grotto formal view must be understood, based on ultrametric spaces ${ }^{21}$. In any case, this last

\footnotetext{
${ }^{18}$ Like in apprehension.

${ }^{19}$ Like in the expressed thought.

${ }^{20}$ Following [44], maybe only Lacan was able to give an original and expressive description of mode of manifestation of the unconscious. Indeed, the elusive-allusive-illusive modality, the incrustations of rhetorical figures, the kaleidoscopic erudition, the intentional ambiguity, the grandiose expression, the perverse echoes of past authors, the oblique irony, the disdain of logical sequence, the humour and sarcasm, are all forms of an affected and precious modality with which Lacan wanted to deliberately show, with his celebrated verbal eloquence, what perverse manners are used by the unconscious to manifest itself.

${ }^{21}$ In this regard, see also what is said in section 5. of the preface, by Remo Bodei, to [45].
}

type of question would deserve further attention ${ }^{22}$. En passant, just for what has been said above about rule changing in transference, we would also want to put forward the hypothesis according to which the celebrated KripkeWittgenstein paradox, inasmuch as it is strictly involved in the critical question around the origins of rules, could receive a useful clarification from the psychoanalytic epistemology standpoint.

\section{CONCLUSIONS}

In this paper, we have mainly discussed, within the general context of the history of culture, the crucial passage from symmetric to asymmetric thinking within the Matte Blanco framework, trying to formalize it through some elementary formal algebraic structures, the groupoids, with respect to which critical comparisons between equivalence and order binary relations have been made possible. Some simple formal considerations have been then put forward, also taking into account some elementary basic notions drawn from theoretical physics. But the landmark point which we wish to highlight in this paper is how order (asymmetry) springs out of that utterly disordered and chaotic realm that is the unconscious. This has been summarized masterfully by the following beautiful Greek maxim due to Anaxagoras of Clazomenæ ${ }^{23}$

$$
\begin{aligned}
& \text { "In the beginning, all the things were together; } \\
& \text { then, it came the mind (ó vov́), and set them in order». }
\end{aligned}
$$

Hence, once again, a great appreciation for the foresight of classical wisdom.

\section{ACKNOWLEDGMENTS}

My warm thanks to Fionn Murtagh. With him, I have been in correspondence on many points regarding his appreciated work on computational psychoanalysis. He has also been very kind in reviewing and correcting this my work. Furthermore, I'm also indebted to all three anonymous reviewers of this paper, whose very useful comments and hints have contributed to improving the present work.

\footnotetext{
${ }^{22}$ In any case, it seems that all these last types of considerations are part of a more general and wider epistemological discussion inherent in the origin, meaning and validity of the contradiction principle, its possible levels, types and interpretations, in the light of the critical relationships between mythological and rational thought.

${ }^{23}$ See, Diogenes Lærtius, Lives of Eminent Philosophers, Volume II, Chapter III.
} 


\section{REFERENCES}

[1] I. Matte Blanco. The Unconscious as Infinite Sets. An Essay in BiLogic. London, UK: Gerald Duckworth, 1975.

[2] L. Figà-Talamanca Dore. La logica dell'inconscio. Introduzione all'opera di Ignacio Matte Blanco. Roma, IT: Edizioni Studium - Vita Nova, 1978.

[3] P.T. Matthews. Introduction to Quantum Mechanics. Maidenhead, Berkshire, UK: McGraw-Hill Publishing Company Limited, 1974.

[4] A. Guay and B. Hepburn, "Symmetry and its Formalisms: Mathematical Aspects", Philosophy of Science, Volume 76, Number 2, pp. 160-178, 2009.

[5] K. Brading and E. Castellani, "Symmetry and Symmetry Breaking", The Stanford Encyclopedia of Philosophy, Spring 2013 Edition, Principal Editor Edward N. Zalta.

[6] L.A. Radicati, "Remarks on the early developments of the notion of symmetry breaking', in: M.G. Doncel, A. Hermann, L. Michel and A. Pais (Eds.). Symmetries in Physics (1600-1980). Proceedings of the $1^{\text {st }}$ International Meeting on the History of Scientific Ideas, Sant Feliu de Guìxois, Catalonia, Spain, September 20-26, 1983, Barcelona, ES: Servei de Publicacions, Bellaterra, 1985, pp. 195-207.

[7] M. Golubitsky and I. Stewart, "Nonlinear dynamics of networks: the groupoid formalism", Bulletin of the American Mathematical Society (New Series), Volume 43, Number 3, pp. 305-364, July 2006.

[8] F. Strocchi, "Spontaneous Symmetry Breaking in Quantum Systems. A review for Scholarpedia", preprint arXiv:1201.5459v1 [physics.hist-ph], 26 January 2012.

[9] F. Strocchi, "Simmetrie e rotture di simmetrie in fisica", Corso di orientamento preuniversitario, Cortona, Arezzo, IT, 3-9 Settembre 2000.

[10] R. Brown, "From Groups to Groupoids: A Brief Survey", Bulletin of the London Mathematical Society, Volume 19, pp. 113-134, 1987.

[11] A. Weinstein, "Groupoids: Unifying Internal and External Symmetry", Notices of the American Mathematical Society, Volume 43, Number 7, pp. 744-752, 1996.

[12] M.R. Buneci, "Topologies on the graph of the equivalence relation associated to a groupoid", Acta Universitatis Apulensis. MathematicsInformatics, Number 6, 2003 [Proceedings of the International Conference on Theory and Applications of Mathematics and Informatics, ICTAMI, Part A, Alba Julia, pp. 23-32, 2003].

[13] F. Harary, Z. Norman and D. Cartwright (Eds.). Structural Models. New York, NY: John Wiley and Sons, Inc, 1965.

[14] F. Latremoliere, "On Quantum Groupoids", preprint available at www.math.berkeley.edu

[15] R.T. Živaljević, Groupoids in combinatorics - applications of a theory of local symmetries, preprint arXiv: math/0605508v1 [math.CO], 18 May 2006.

[16] G. Barthe, V. Capretta and O. Pons, "Setoids in type theory", Journal of Functional Programming, Volume 13, Issue 2, pp. 261-293, March 2003.

[17] N.D. Gilbert and E.C. Miller, "The graph expansion of an ordered groupoid", Algebra Colloquium, Volume 18, Issue 1, pp. 827-842, December 2011 .

[18] G. Iurato, "Mathematical thought in the light of Matte Blanco's work", Philosophy of Mathematics Education Journal, Volume 27, pp. 1-8, April 2013.

[19] M.K. Lawson, "Constructing ordered groupoids", Cahiers de Topologie et Géométrie Différentielle Catégoriques, Volume 46, Number 2, pp. 123-138, 2005.

[20] R. Hermann. Lie Groups for Physicists. New York, NY: W.A. Benjamin, Inc., 1968.

[21] D.R. White and P. Jorion, "Kinship networks and discrete structure theory: applications and implications", Social Networks, Volume 18, pp. 267-314, 1996.

[22] J.G. Kemeny, J.L. Snell and G.L. Thompson. Introduction to Finite Mathematics. 3rd edition, Englewood Cliffs, NJ: Prentice-Hall, 1974.

[23] D. Lerner (Ed.). Quality and Quantity. New York, NY: The Free Press of Glencoe, 1961.
[24] R.L. Atkinson, R.C. Atkinson, E.E. Smith, D.J. Bem and S. NolenHoeksema (Eds.). Hilgard's Introduction to Psychology. 12th edition, Orlando, FL: Harcourt Brace, 1996.

[25] C. Lévi-Strauss. Razza e storia e altri studi di antropologia. Torino, IT: Giulio Einaudi editore, 1975.

[26] G. Bateson. Steps to an Ecology of Mind. Collected Essays in Anthropology, Psychiatry, Evolution, and Epistemology. San Francisco, CA: Chandler Publishing Company, 1972.

[27] W.S. McCulloch. Embodiments of Mind. Cambridge, MA: The MIT Press, 1965

[28] P. Watzlawick, J.H. Beavin, D.D. Jackson. Pragmatics of Human Communication. A Study of Interactional Patterns, Pathologies, and Paradoxes. New York, NY: W.W. Norton, 1967.

[29] G. Iurato, "On Jacques Lacan Psychosis Theory and ERPs Analysis", 2012, preprint No. hal-00750049 version 1, available at HAL archivesouvertes.

[30] D.D. Jackson, "Some factors influencing the CEdipus Complex", Psychoanalytic Quarterly, Volume 23, pp. 566-581, 1954.

[31] M.O. Albertson and K.L. Collins, "Symmetry Breaking in Graphs", The Electronic Journal of Combinatorics, Volume 3, Number 1, R18, 1996.

[32] M. Field. Symmetry Breaking for Compact Lie Groups, Memoir of the AMS No. 574. Providence, RI: American Mathematical Society Publications, 1996.

[33] K. Fink, "The Bi-Logic Perception of Time", International Journal of Psycho-Analysis, Volume 74, pp. 303-312, 1993.

[34] G.B. Vicario, "Il tempo in psicologia", Le Scienze, Volume 30, Number 347, pp. 43-51, July 1997.

[35] J.R. Rose (Ed.). Mapping Psychic Reality: Triangulation, Communication, and Insight. Psychoanalytic Ideas, London, UK: Karnac Books, 2011.

[36] M. Piattelli Palmarini. Scienza come cultura. Protagonisti, luoghi e idee delle scienze contemporanee. Milano, IT: Arnoldo Mondadori Editore, 1987.

[37] C. Wetterich, "Spontaneous Symmetry Breaking Origins for the Difference Between Time and Space", Physics Review Letters, Volume 94, Number 1, pp. 011692-6, 2005.

[38] J. Ellis, N.E. Mavromatos and D.V. Nanopoulos, "The origin of spacetime as W-symmetry breaking in string theory", Physics Letters B, Volume 288, Numbers 1-2, pp. 23-30, 1992.

[39] R. Lauro-Grotto, "The Unconscious as an Ultrametric Set", American Imago, Volume 64, Number 4, pp. 535-543, 2008.

[40] F. Murtagh, "Ultrametric Model of Mind, I: Review”, P-Adic Numbers, Ultrametric Analysis, and Applications, Volume 4, Number 3, pp. 193 206, July 2012.

[41] A.Yu. Khrennikov, "Toward an adequate mathematical model of mental space: conscious/unconscious dynamics on m-adic trees", Biosystems, Volume 90, Number 3, pp. 656-675, 2007.

[42] A.Yu. Khrennikov, 'Human subconscious as the p-adic dynamical system', Journal of Theoretical Biology, Volume 193, pp. 179-196, 1998.

[43] A.Yu. Khrennikov, Classical and quantum mental models and Freud's theory of unconscious mind. Series in Mathematical Modelling in Physics, Enginnering and Cognitive Sciences, Volume 1, Växjö, SE: Växjö University Press, 2002.

[44] S.A. Mitchell and M.J. Black, Freud and beyond. A History of Modern Psychoanalysic Thought. New York, NY: Basic Books. A Division of Harper Collins Publishers, 1995.

[45] I. Matte Blanco. L'inconscio come insiemi infiniti. Saggio sulla bilogica. Nuova edizione a cura di Pietro Bria con prefazione di Remo Bodei. Torino, IT: Giulio Einaudi editore, 2000.

[46] O. Bonnota, M. de Montalembertb, S. Kermarrecc, M. Botbold, M. Waltere and N. Coulon, 'Are impairments of time perception in schizophrenia a neglected phenomenon?', Journal of Physiology-Paris, Volume 105, Issues 4-6, pp. 164-169, December 2011. 